\title{
Thanks to the Reviewers of the Neurointervention
}

\author{
Sang Hyun Suh, MD, PhD \\ Editor-in-Chief, Neurointervention, Seoul, Korea
}

The editors of the Neurointervention thank manuscript reviewers who completed their reviews for the journal for 2021. We sincerely express our gratitude to all the reviewers listed below for taking the time to review with their expertise.

\begin{tabular}{|c|c|c|}
\hline Akira Ishii & Allan Taylor & Anchalee Churojana \\
\hline Anderson Chun On Tsang & Bum Joon Kim & Byung Hyun Baek \\
\hline Chang-Woo Ryu & Cheolkyu Jung & Chul-Ho Sohn \\
\hline Chung-Wei Lee & Deok Hee Lee & Dong Hyun Yoo \\
\hline Dong Woo Park & Dong-Hyun Shim & Dongwhane Lee \\
\hline Dongyoung Cho & Eung Yeop Kim & Gwynedd Pickett \\
\hline Hae Woong Jeong & Hiro Kiyosue & Hong Gee Roh \\
\hline Hon-Man Liu & Hyo Sung Kwak & Hyun Jeong Kim \\
\hline Hyung Jong Park & Hyun-Seung Kang & Isil Saatci \\
\hline Jae Jon Sheen & Jai Ho Choi & Jianmin Liu \\
\hline Jieun Roh & Jin Pyeong Jeon & Jin Soo Lee \\
\hline Jin Woo Choi & Jin Wook Choi & Jinhee Jang \\
\hline Jong S. Kim & Jong-Ho Park & Joonggoo Kim \\
\hline Joonho Chung & Jun Yup Kim & Junhyung Kim \\
\hline Keun Young Park & Kwon-Duk Seo & Kyung Sik Yi \\
\hline Marie-Christine Brunet & Michael Söderman & Michihiro Tanaka \\
\hline Minkyu Han & Myong Jin Kang & Myung Ho Rho \\
\hline Naci Kocer & Pyoung Jeon & René Chapot \\
\hline René van den Berg & Ronit Agid & Saima Ahmad \\
\hline Samyeol Ha & Sang Hun Lee & Sang Hyun Suh \\
\hline Sangheum Kim & Sedat Giray Kandemirli & Seung Kug Baik \\
\hline Shigeru Miyachi & So Yeon Kim & Sung Hyun Baik \\
\hline Sung-Tae Park & Tae Hong Lee & Winston Chong \\
\hline Wong Kwok Chu & Woo Sang Jung & Woong Yoon \\
\hline Yon-Kwon Ihn & Yoonkyung Chang & Young Dae Cho \\
\hline ung-Jun Lee & & \\
\hline
\end{tabular}

\section{Correspondence to:} Sang Hyun Suh, MD, PhD

Department of Radiology, Gangnam Severance Hospital, Yonsei University College of Medicine, 211 Eonju-ro, Gangnam-gu, Seoul 06273, Korea Tel: +82-2-2019-3510

Fax: +82-2-3462-5472

E-mail: suhsh11@yuhs.ac
Copyright $\odot 2022$ Korean Society of Interventional Neuroradiology

This is an Open Access article distributed under the terms of the Creative Commons Attribution Non-Commercial License (http://creativecommons.org/licenses/by-nc/4.0) which permits unrestricted non-commercial use, distribution, and reproduction in any medium, provided the original work is properly cited. 